\section{Síndrome de piernas inquietas (enfermedad de Willis-Ekbom): seguimiento a largo plazo de una serie de pacientes}

\author{
MARCELO MIRANDA C., LORENA HUDSON A.
}

\section{Restless legs syndrome: long-term follow-up of a series of patients}

Background: Restless legs syndrome (RLS) affects 10\% of the general population. Aim: To analyze a series of patients with a minimum follow-up period of four years, treated during an interval of 14 years. Material and Methods: Retrospective analysis of medical records of 200 patients assessed and followed by the authors at a private outpatient clinic. Results: Fifty patients aged 25 to 90 years (34 females), who had a mean follow-up of 6,3 years (range 4-14 years), were selected. Sixty percent responded to therapy that initially consisted in dopamine agonists in $78 \%$ of cases. Thirty four percent remained symptomatic and $4 \%$ worsened. RLS severity scale improved from an initial score of 19,2 to 12,5 at the last follow-up visit $(p<0.05)$. Thirty-three patients $(66 \%)$ experienced an overall worsening of symptoms beyond pretreatment levels during follow-up. The strategies to overcome this augmentation were the change to another agonist, use of ligands such as pregabalin and gabapentin, opioids and iron. Low ferritin was common in most of the patients in whom it was measured (24 of 45 results), mainly in those with augmentation $(p<0,05)$. Six percent of patients treated with dopamine agonist developed an impulse control disorder. Conclusions: RLS is a treatable condition during a long period of follow-up in most patients. We found a high rate of potentiation at presentation which can be explained by the inadequate use of high doses of dopaminergic agents.

(Rev Med Chile 2016; 144: 1561-1566)

Key words: Dopamine Agents; Dopamine Agonists; Drug Synergism; LongTerm Potentiating; Restless Legs Syndrome.

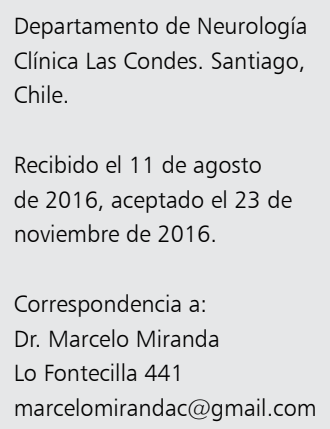


y los llamados agonistas de la dopamina como pramipexole, ropinerole, rotigotine ${ }^{5}$. Este síndrome es conocido también como enfermedad de Willis-Ekbom, por ser Thomas Willis, en el siglo XVII, quien hizo la primera descripción histórica y la primera terapia en base a láudano; luego Karl Ekbom, en el siglo XX, lo definió más claramente como una entidad nosológica ${ }^{3}$.

Existe una forma idiopática de SPI y otra secundaria a patologías crónicas, destacando, especialmente por su severidad, la de los pacientes urémicos en diálisis $(20-30 \%)^{2}$.

También es frecuente en diabéticos, generalmente asociado a neuropatía, anemia, deficiencia de hierro con o sin anemia, y a otras polineuropatías ${ }^{1,3}$. También se observa en condiciones fisiológicas como el embarazo (12-30\%), atribuyéndose en estos casos a una deficiencia de hierro y ácido fólico ${ }^{1,3}$.

Existen pocos estudios sobre la evolución clínica de este cuadro y del seguimiento a largo plazo de los pacientes tratados o no ${ }^{2,6-10}$, ninguno de ellos en Latinoamérica. En el estudio más importante de seguimiento publicado hasta ahora, Mitterlinck y cols describen una mejoría de los síntomas en $55 \%$ de los pacientes tratados seguidos a 5 años, sin embargo, $45 \%$ permanecía igual o peor ${ }^{2}$. Un estudio en Japón mostró que a 2 años sólo 50\% de los pacientes diagnosticados inicialmente (y no tratados) seguían sintomáticos ${ }^{10}$, lo que nos dice que en algunas poblaciones hay una alta remisión espontánea.

Tener información sobre la evolución clínica y respuesta a la terapia en nuestro medio nos ayudará a un mejor manejo farmacológico inicial del cuadro, con el fin de intentar evitar una de sus complicaciones más importantes, como es el fenómeno de potenciación, que llega a afectar a entre 20 y $80 \%$ de los pacientes tratados. La potenciación consiste en agravación de la sintomatología, con inicio más precoz de los síntomas (en la tarde), con menor latencia de aparición al estar en reposo, compromiso de otros segmentos corporales como extremidades superiores y tronco, mayor severidad de síntomas y más breve duración de efecto de la terapia ${ }^{11-13}$. Un factor en su desarrollo es que el paciente tenga niveles bajos de ferritina al inicio de la terapia y sea tratado con dosis de dopaminérgicos mayores a lo recomendado ${ }^{11-13}$.

El objetivo de esta revisión de una serie clínica es describir la evolución clínica de los pacientes con SPI a través del seguimiento durante varios años y los efectos de la terapia.

\section{Pacientes y Método}

Estudio observacional, descriptivo de una serie clínica retrospectiva, de los pacientes atendidos por los autores en el Departamento de Neurología de Clínica Las Condes desde los años 2001 al 2016. Se incluyeron pacientes que cumplieran criterios diagnósticos para SPI propuestos por el Grupo Internacional de Estudios en piernas inquietas $\left(\right.$ GIEPI ${ }^{1}$ y que son: 1 ) imperiosa necesidad de mover las piernas asociada a sensaciones desagradables en extremidades; 2) ocurre en reposo de preferencia en la noche, y 3 ) característicamente se alivia moviendo las extremidades afectadas. Además, como criterio de inclusión se consideraron sólo los que tuvieron un seguimiento de a lo menos cuatro años y con más de 2 visitas de control en ese período.

La severidad del cuadro fue determinada con la escala de severidad del SPI propuesta por el GIEPI $^{1}$, que mide la severidad con 10 parámetros, como son: intensidad de síntomas, necesidad de moverse, alivio obtenido, alteración del sueño, cansancio derivado de estas molestias, frecuencia semanal, duración diaria, interferencia con actividades diarias y consecuencias en el ánimo. Cada uno de estos ítems se cuantifica de 0 (nunca) a 4 (muy severo), lo que estratifica cinco niveles de severidad: Muy severo: 31-40 puntos, severo 2130, moderado 11-20, leve: 1-10, ausente: 0 .

Se describieron también las siguientes variables: necesidad de terapia, tipo de fármacos, respuesta terapéutica, complicaciones de la terapia y manejo de ésta, así como influencia de los niveles de ferritina en la respuesta clínica. Se consideró niveles bajos de ferritina a valores menores de 75 $\mathrm{mg} / \mathrm{dl}$, de acuerdo a lo sugerido por el GIEPI ${ }^{5}$.

Se definió respuesta terapéutica como cambios en al menos un nivel en el grado de severidad (por ejemplo, pasar de nivel moderado a leve).

Los pacientes dieron su consentimiento escrito para el uso de su información clínica y el estudio fue aprobado por el Comité de Ética local.

Análisis estadístico: El análisis estadístico fue realizado usando el software SPSS para Windows versión 24.0. Los datos se presentan en promedios (rangos) para las variables cuantitativas, $o$ frecuencias, para variables cualitativos, según co- 
rresponda. Se evaluó la normalidad con la prueba de Kolmogorov-Smirnov. Las comparaciones de grupo para variables cuantitativas se efectuaron por medio de la prueba de Wilcoxon, para variables independientes o relacionadas según corresponda. Las comparaciones de grupo para variables cualitativas se efectuaron por medio de la prueba de $\chi^{2}$. Para todos los tests se usó un nivel de significancia de 0,05 .

\section{Resultados}

De un total de 200 pacientes vistos por diagnóstico de SPI en 15 años, se seleccionaron 50 pacientes que cumplieron criterios de inclusión de tener más de una segunda visita al cabo de al menos 4 años de control. Todos los pacientes tuvieron la forma idiopática de la enfermedad. Veinticinco (50\%) comunicaron tener al menos 1 familiar afectado. Treinta y cuatro eran mujeres (68\%) y 16 hombres; la edad promedio fue 56,4 años (rango 25-90); años de duración de síntomas: 12,7 (rango 4-40); años de seguimiento promedio de 6,3 (rango 4-14). Cuarenta y tres pacientes (86\%) tuvieron un seguimiento de 5 años o más.

El rango severidad en primera evaluación mostró un puntaje promedio de todos los pacientes de 19,2 y al cabo de 4 años de 12 puntos $(\mathrm{p}<0,05)$ (Figura 1). Cuarenta y siete de los 50 casos (94\%) estaba en el rango de severidad de tipo moderado a muy severo, mientras que al cabo de segunda visita, 27 pacientes (54\%) estaba en este rango de severidad $(\mathrm{p}<0,05)$.

Treinta $(60 \%)$ de los 50 pacientes mejoraron en síntomas, de 21 puntos promedio inicialmente pasaron a 10 puntos al cabo de 4 años $(\mathrm{p}<0,05)$. Diecisiete $(34 \%)$ se mantuvieron sin variación y $3(6 \%)$ empeoran.

Veinte de los 50 pacientes (40\%) se presentaron con síntomas de potenciación en primera visita: ellos eran los únicos que estaban siendo tratados al momento de presentarse a primera consulta. Recibían dosis más altas de lo recomendado de agonistas ( 10 con pramipexole dosis hasta $1,5 \mathrm{mg}$ día, 5 con ropinerole, hasta $6 \mathrm{mg}$ día y levodopa, 5 casos con dosis hasta $1 \mathrm{~g}$ día).

En 3 pacientes pudo observarse síntomas del síndrome de descontrol de impulsos provocados por los agonistas dopaminérgicos (juego patológico, compras compulsivas); cedió la sintomatología con la reducción de la dosis.

Al cabo de la visita de seguimiento hubo potenciación en 33 casos (66\%), 21 de ellos logró mejorar con el ajuste de fármacos.

La terapia inicial, luego de primera consulta (Tabla 1), consistió en agonistas de la dopamina como pramipexole o ropinerole en 38 casos y 1 caso en parche de rotigotine; 6 casos recibieron

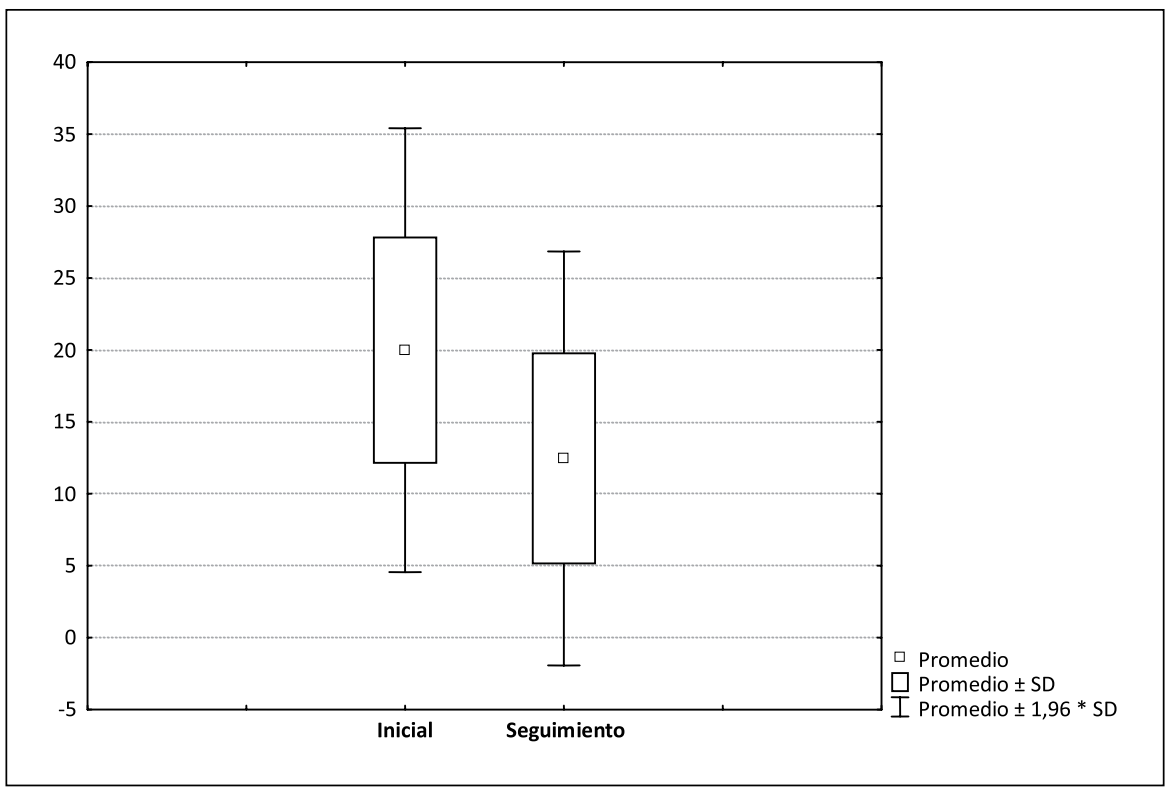

Figura 1. Puntaje en la escala de severidad de síntomas de piernas inquietas en visita inicial y al seguimiento 
Tabla 1. Frecuencias de uso de fármacos al inicio y en el seguimiento*

\begin{tabular}{|lrrrr|}
\hline & $\mathbf{n}$ & $\mathbf{( \% )}$ & $\mathbf{n}$ & $\mathbf{( \% )}$ \\
\hline Levodopa & 6 & $(12)$ & \multicolumn{2}{c|}{-} \\
Agonistas & 39 & $(78)$ & 29 & $(58)$ \\
Ligando $\alpha 2 \delta$ & 3 & $(6)$ & $25^{* *}$ & $(50)$ \\
Opioides & & - & 3 & $(6)$ \\
\hline
\end{tabular}

*Hierro se usó en 24 casos como coadyudante. **ligandos $\alpha 2 \delta$ se asociaron en 10 casos a agonistas y en 3 a opiodes.

levodopa. En 24 casos se usó hierro como coadyudante, 3 casos con ligandos $\alpha 2 \delta$, como pregabalina o gabapentina. Un paciente se mantuvo sin terapia al cabo del seguimiento.

Al final del seguimiento (Tabla 1), la terapia consistió en 16 pacientes con pramipexole o ropinerole, 13 con rotigotine, 15 con pregabalina y 10 con gabapentina. Diez pacientes usaron una combinación de agonistas asociado a pregabalina o gabapentina. Opioides (oxicontina) se usó en 3 casos y, finalmente, hierro como coadyuvante en 24 pacientes. Dos pacientes que tuvieron remisión de síntomas no siguieron con fármacos.

Se obtuvo valores de ferritina en 45 casos, en aquellos pacientes que tenían aumentación los niveles de ferritina fueron significativamente más bajos que los que no tuvieron potenciación $(\mathrm{p}<0,05)$. No se encontró relación en la respuesta terapéutica respecto a sexo, edad o duración de síntomas.

En la terapia se puede observar que para el manejo de los casos de potenciación se realizó una modificación en la terapia que consistió, básicamente, en reducción de dosis de dopaminérgicos, cambio a otro agente dopaminérgico o uso de otro agente tipo ligandos $\alpha 2 \delta$ como pregabalina o gabapentina, o uso de opioides (oxicontina en 3 casos) (Tabla 2).

\section{Discusión}

Este es el primer estudio en nuestro medio de evaluación de la terapia a largo plazo en una serie de pacientes con SPI. Tiene la limitación de ser una evaluación retrospectiva y que considera finalmente sólo $25 \%$ del total de pacientes vistos y que son los que cumplen con criterios de inclusión. A pesar de esta limitante, los resultados
Tabla 2. Cambios de medicamentos más frecuentes en seguimiento

\begin{tabular}{lrr|}
\hline & $\mathbf{n}$ & $\mathbf{( \% )}$ \\
Levodopa $\rightarrow$ agonista & 6 & $(12)$ \\
Agonista $\rightarrow$ agonista & 18 & $(36)$ \\
Agonista $\rightarrow$ ligando $\alpha 2 \delta$ & 20 & $(40)$ \\
Agonista $\rightarrow$ opioides & 3 & $(6)$ \\
Agonista $\rightarrow$ nada & 3 & $(6)$ \\
\hline
\end{tabular}

muestran que el SPI es un cuadro manejable en la mayoría de los pacientes (60\%), lo que está de acuerdo con series publicadas, como las de Mitterling et al, que encuentran mejoría en $55 \%$ de 160 casos seguidos. El porcentaje de pacientes que no mejora o se mantiene igual (34\%) está también en concordancia con la literatura ${ }^{2,5,8,9}$.

Los agentes dopaminérgicos han sido usados en el tratamiento de SPI por más de 30 años ${ }^{5}$. Su eficacia a largo plazo ha sido bien demostrada ${ }^{5}$. Los efectos secundarios son leves y transitorios ${ }^{5} y$ en esta condición no se observan las disquinesias que se frecuentemente ocurren en la enfermedad de Parkinson. Sin embargo, sí se observa el síndrome de descontrol de impulsos (SDI) provocado por la terapia, como sucede en la enfermedad de Parkinson ${ }^{14}$. Se describe su presencia entre 6 y $17 \%$ de los pacientes tratados con agonistas por SPI ${ }^{15}$. En esta serie observamos en 3 pacientes (6\%) el SDI que puedo manejarse con reducción de la dosis del agonista.

La mayor complicación es la pérdida de eficacia de la terapia y agravación de los síntomas inducida por ella, lo que se conoce como potenciación. A diferencia de la enfermedad de Parkinson, subir la medicación, sólo empeorara los síntomas. Si no existe conocimiento de este efecto secundario, el médico tenderá a subir más y más la medicación lo que empeorará al paciente y será un círculo vicioso: es como lo que sucede, por ejemplo, con la cefalea por abuso de analgésicos.

De acuerdo con las guías actuales de terapia $\mathrm{SPI}^{5}$ podemos ver un claro predominio de agentes dopaminérgicos en la primera etapa de tratamiento de esta serie de pacientes, en comparación con la última visita analizada. Así, en el seguimiento hay un claro aumento de agentes no dopaminérgicos como pregabalina, gabapentina u 
opioides lo que refleja la limitación a largo plazo de esta terapia y la actual disponibilidad de nuevas alternativas de manejo SPI ${ }^{16,17}$. En ese sentido, el uso de opioides es una buena opción, ya que no induce potenciación ${ }^{2,16}$, salvo tramadol ${ }^{5}$ y se ha visto que el riesgo de adicción en SPI es bajo en las dosis habitualmente usadas, como promedio 20 mg día en nuestros casos (10-70/mg día en series publicadas $)^{2,14}$. La dificultad para prescribirlo por el tipo de receta restringida limita su uso y explica los pocos casos en esta serie que lo usaron, pero en los que fue muy útil y también en series publicadas que no alcanza el 13\% de los casos tratados.

Es llamativo el alto porcentaje de potenciación al momento de la primera consulta de los pacientes, tal como se comentó en una comunicación anterior sobre estos $\operatorname{casos}^{11}$ : todos estaban siendo tratados con dosis más altas de la recomendada para tratar SPI. Es probable que también refleje un sesgo de referencia: desde que se hizo énfasis en nuestro medio de lo prevalente que es el SPI ${ }^{3}$ y la difusión en los medios, son los pacientes más severos los que tienden a consultar. Así es como $94 \%$ estaba en el rango de moderado a muy severo, sólo 3 pacientes tenían síntomas leves y sólo 1 se mantuvo sin terapia al cabo del período de seguimiento. En otras series publicadas, llama la atención que $20 \%$ de los pacientes se mantienen sin terapia al cabo de un período de seguimiento ${ }^{2}$, en comparación con sólo 3 casos (6\%) en esta serie que no requiere terapia en control a largo plazo. Lo que refuerza lo ya señalado en términos que se trata de una muestra de casos de SPI severo.

La dosis recomendada para tratar SPI son menos de $0,75 \mathrm{mg}$ de pramipexole, menos de $4 \mathrm{mg}$ de ropinerole, menos de $200 \mathrm{mg}$ levodopa. La corrección de factores de riesgo como baja ferritina, reducción de dosis de dopaminérgicos, cambio de terapia a otro tipo de agonista o de presentación en parche con efecto continuo (rotigotine), el uso de agentes no dopaminérgicos como pregabalina o gabapentina, como también de opioides, logró en estos pacientes un adecuado manejo en 21 de 33 casos que presentaban potenciación al cabo de 4 años. En este sentido, se reafirma el rol de hierro en la fisiopatología de este trastorno y específicamente de niveles bajos ferritina ${ }^{4}$ : de 45 casos en que se estudió los niveles de ferritina, estuvo baja en 24 casos, siendo 18 de ellos con síntomas de potenciación.

Las medidas preventivas más importantes para evitar complicaciones en la terapia de SPI son el uso y mantención de dosis bajas de agentes dopaminérgicos y lograr niveles de ferritina sobre $75 \mathrm{mg} / \mathrm{dl}^{5}$.

En suma, se concluye que en el seguimiento de pacientes con SPI, la mayoría tiene una buena respuesta a la terapia y los que presentan complicaciones del tratamiento pueden ser, en su mayoría, manejados adecuadamente. Es necesario un mejor conocimiento en nuestro medio sobre el manejo de este prevalente trastorno en la práctica clínica.

\section{Referencias}

1. Allen RP, Picchietti D, Hening WA, Trenkwalder C, Walters AS, Montplaisir. Restless legs syndrome: diagnostic criteria, Sleep Med 2003; 4: 101-19.

2. Mitterling T, Heidbreder A, Stefani A, Fritz J, Ulmer H, Poewe W, et al. Natural course of restless legs syndrome/ Willis-Ekbom disease: long-term observation of a large clinical cohort. Sleep Med 2015; 1252-8.

3. Miranda M, Araya F, Castillo JL, Durán C, González F, Arís L. Síndrome de piernas inquietas: Estudio clínico en población general adulta y en pacientes urémicos Rev Med Chile 2001; 129: 179-86.

4. Allen R. Dopamine and iron in the pathophysiology of restless legs syndrome (RLS). Sleep Med 2004; 5: 385-91.

5. García-Borreguero D, Kohnen R, Silber MH. The longterm treatment of restless legs syndrome/Willis-Ekbom disease: evidence-based guidelines and clinical consensus best practice guidance: a report from the International Restless Legs Syndrome Study Group. Sleep Med 2013; 14: 675-84.

6. Szentkiralyi A, Fendrich K, Hoffmann W. Incidence of restless legs syndrome in two population-based cohort studies in Germany. Sleep Med 2011; 12: 815-20.

7. Fuhs A, Bentama D, Antkowiak R. Effects of short- and long-term variations in RLS severity on perceived health status-the COR Study. PLoS ONE 2014; 9 (4): e9482 doi:10.1371/journal.pone.0094821.

8. Silver N, Allen RP, Senerth J. A 10-year, longitudinal assessment of dopamine agonists and methadone in the treatment of restless legs syndrome. Sleep Med 2011; 12: 440-4.

9. Lipford MC, Silber MH. Long-term use of pramipexole in the management of restless legs syndrome. Sleep Med 2012; 13: 1280-5.

10. Kagimura T, Nomura T, Kusumi M. Prospective survey on the natural course of restless legs syndrome over two years in a closed cohort. Sleep Med 2011; 12: 821-6.

11. Miranda CM. Rotigotine transdérmico en el manejo 
del síndrome de piernas inquietas en pacientes con potenciación de los síntomas debido a la terapia dopaminérgica. Rev Med Chile 2013; 141: 407-8.

12. García-Borreguero D, Williams AM. Dopaminergic augmentation of restless legs syndrome. Sleep Med Rev 2010; 14: 339-46.

13. García-Borreguero D, Allen RP, Kohnen R, Hogl B, Trenkwalder C, Oertel W. Diagnostic standards for dopaminergic augmentation of restless legs syndrome: report from a World Association of Sleep MedicineInternational Restless Legs Syndrome Study Group consensus conference at the Max Planck Institute. Sleep Med 2007; 8: 520-3.

14. Miranda M, Bustamante L, Pérez C. Juego patológico como efecto secundario del tratamiento de la enfermedad de Parkinson. Rev Med Chile 2010; 138: 521-2.

15. Cornelius JR, Tippmann-Peikert M, Slocumb NL, Frerichs CF, Silber MH. Impulse control disorders with the use of dopaminergic agents in restless legs syndrome: a case-control study. Sleep 2010; 33: 81-7.

16. Trenkwalder C, Benes H, Grote L. Prolonged release oxycodone-naloxone for treatment of severe restless legs syndrome after failure of previous treatment: a double-blind, randomised, placebo-controlled trial with an open-label extension. Lancet Neurol 2013; 12: 1141-50.

17. Allen RP, Chen C, García-Borreguero D. Comparison of pregabalin with pramipexole for restless legs syndrome. N Engl J Med 2014; 370: 621-31.

\section{ARTE Y FOTOGRAFÍA}

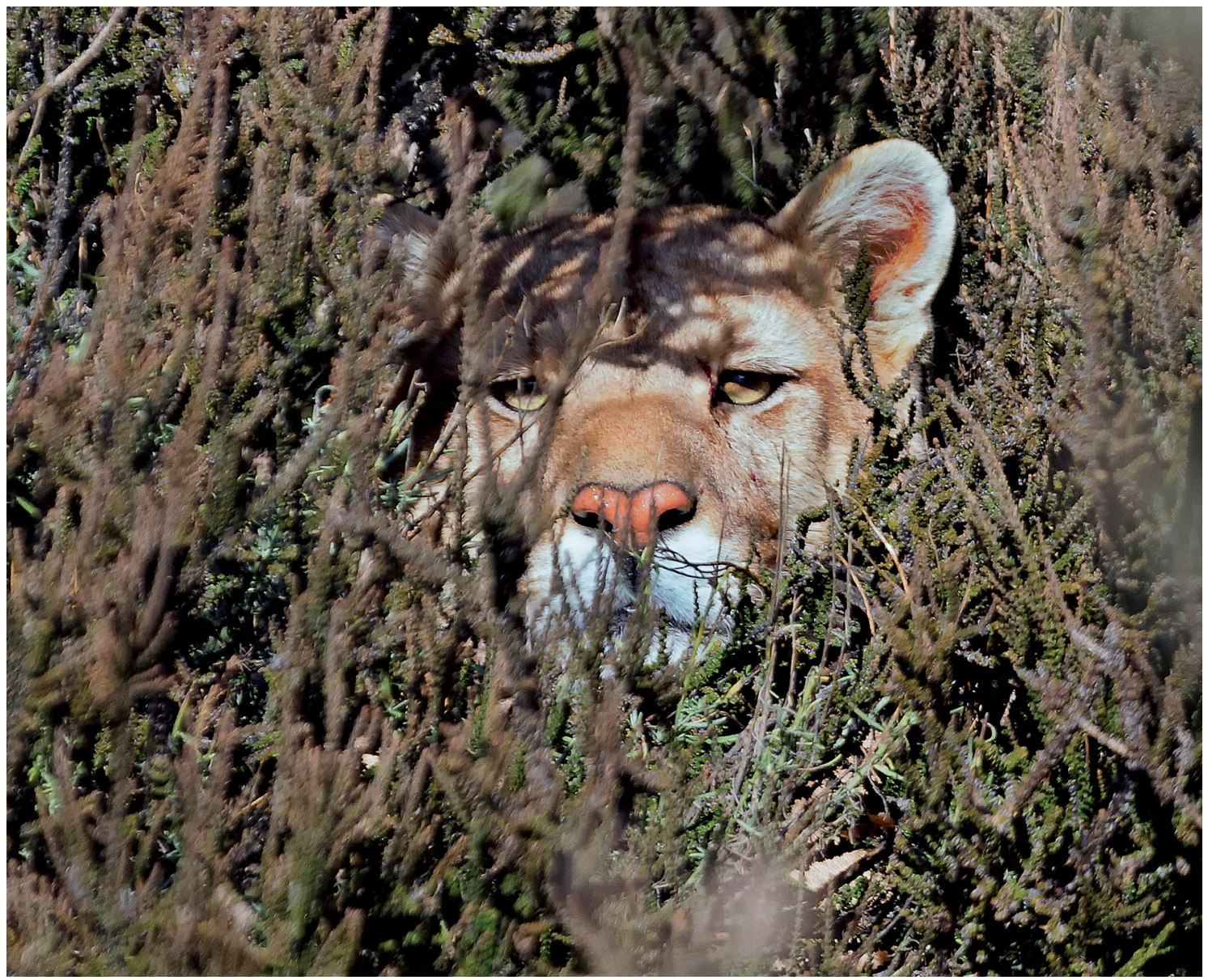

Puma, Torres del Paine. Dr. Marcos Skarmeta Muranda 